\title{
Análise do acolhimento de crianças e adolescentes para o planejamento das ações do PSF
}

\author{
Analysis of drop-in visits of children and adolescents \\ for planning the actions of the Family Health Program
}

\author{
Cláudia Regina Lindgren Alves ${ }^{1}$ \\ Jaciara Lagazeta Garcia ${ }^{2}$ \\ Carmen Cadete Gomes Silveira ${ }^{3}$ \\ Gustavo Vieira Rodrigues Maciel ${ }^{4}$ \\ Maria Teresa Soares Souza ${ }^{4}$
}

\begin{abstract}
Resumo
O atendimento à demanda não-programada (acolhimento) é hoje um dos mais importantes desafios da atenção básica. Dimensioná-lo representa um dos pontos de partida para a organização do trabalho das equipes de saúde da família (ESF). Este estudo teve por objetivo analisar a demanda não-programada de crianças e adolescentes de uma das ESF do Centro de Saúde São Marcos (CSSM), visando ao planejamento de ações. O acolhimento no CSSM, localizado na região nordeste de Belo Horizonte, é realizado pela ESF durante todo o dia. Foram analisados os registros do atendimento à demanda não-programada em um determinado horário do dia, reservado para este fim, no período de outubro de 2005 a setembro de 2006. A amostra foi composta por 30\% dos cerca de cinco mil atendimentos realizados, sorteados aleatoriamente. O banco de dados foi analisado no Epi-Info. Foram analisados 1602 atendimentos, dos quais $627(39,2 \%)$ eram menores de 20 anos de idade. Cerca de $24 \%$ eram crianças menores de dois anos e $26 \%$ eram adolescentes. Os pacientes com queixas agudas corresponderam a 80,4\% dos atendimentos em toda faixa pediátrica. Pouco mais da metade das crianças eram do sexo feminino. Cerca de 100 crianças (16,9\%) procuraram o acolhimento para agendar consultas e/ou mostrar exames. Dessas, $12 \%$ eram pacientes de puericultura e 5\% eram portadores de problemas respiratórios crônicos, como asma. Quatro adolescentes procuraram o acolhimento para fazer o pré-natal. O pequeno percentual de crianças menores de dois anos no acolhimento sugere que o fluxo de pacientes no controle/ puericultura mensal tem sido eficaz. O mesmo pode ser dito das crianças inscritas no programa Criança que Chia. No entanto, o grande percentual de crianças até 10 anos com queixas agudas requer planejamento da agenda para atendê-las o mais rapidamente possível. Com base nesses resultados, pretende-se reorganizar as agendas e rever as atribuições dos profissionais da equipe, propondo alternativas de atenção ao usuário dessa faixa etária.
\end{abstract}

Palavras-chave: Acolhimento; Planejamento em Saúde; Atenção Primária à Saúde; Programa de Saúde da Família.
Key Words: User embracement; Health Planning;

Primary Health Care; Family Health Program.

${ }^{1}$ Professora, doutora em Ciências da Saúde - área de concentração em saúde da criança e do adolescente, Departamento de Pediatria, Faculdade de Medicina, Universidade Federal de Minas Gerais, Belo Horizonte, Brasil.

${ }^{2}$ Enfermeira, especialista em Saúde da Família pela UFMG, Secretaria Municipal de Saúde, Belo Horizonte, Brasil.

${ }^{3}$ Gerente do Centro de Saúde São Marcos, especialista em Enfermagem na Saúde Pública pela UFMG, Secretaria Municipal de Saúde, Belo Horizonte, Brasil.

${ }^{4}$ Acadêmico(a) do curso de graduação em medicina da Universidade Federal de Minas Gerais, Belo Horizonte, Brasil. 


\begin{abstract}
Today, establishing a drop-in policy is one of the most important challenges of primary care. Find the right dimension for delivering care on this basis is one of the starting points for organizing the work of the Family Health Teams (FHT). The purpose of this study was analyzing the demand of drop-in visits of children and adolescents of one of the Family Health Teams of the São Marcos
\end{abstract} Health Center (SMHC) for planning the actions of the team. The family bealth team of the SMHC, situated in the Northeast of the city of Belo Horizonte, accepts drop-in visits during all day. The medical records over the period October 2005-September 2006 corresponding to the drop-in visits during a certain period of the day, which was reserved for this activity, were analyzed. The sample consisted of $30 \%$ of the about 5.000 visits, selected at random. The data were analyzed using Epi-Info. From the 1602 analyzed visits, 627 (39,2\%) were of patients with less than 20 years of age. About 24\% were children with less than 2 years and $26 \%$ were adolescents. The patients with acute complaints corresponded to 80,4\% of all pediatric visits. A little more than half of the children were of feminine sex. Approximately 100 children (16,9\%) dropped in for making appointments or for showing the results of exams. From these, 12\% were well-child care cases and 5\% carriers of chronic respiratory diseases like asthma. Four adolescents came for pre-natal care. The low percentage of children with less than two years of age in the dropin visits suggests that the monthly well-child care had worked efficiently. The same refers to the children inscribed in the asthma treatment program "Criança que Chia". However, most of the children up to 10 years with acute complaints need appointments so that they can be cared for as quickly as possible. On the basis of these results the agendas will be reorganized and the attributions of the team members will be reviewed proposing alternatives for the users of this age group.

\section{Introdução}

O termo acolhimento vem sendo amplamente discutido nos diversos cenários do cuidado com a saúde da população e tem adquirido diferentes significados entre os profissionais, os usuários e os gestores dos serviços. Dentro das propostas para humanização da Atenção Básica do Ministério da Saúde, acolhimento significa "recepção do usuário, desde sua chegada, responsabilizando-se integralmente por ele, ouvindo sua queixa, permitindo que ele expresse suas preocupações, angústias e, ao mesmo tempo, colocando os limites necessários, garantindo atenção resolutiva e a articulação com outros serviços de saúde para a continuidade da assistência"

Em Belo Horizonte, o acolhimento foi implementado na rede básica de saúde a partir de 1994, buscando humanizar a atenção, estabelecer vínculo entre os usuários e o serviço, além de aumentar a capacidade de escuta às demandas apresentadas, ampliando as intervenções e a responsabilização das equipes com os usuários ${ }^{2}$.

O Programa de Saúde da Família (PSF) no Município de Belo Horizonte foi implantado em fevereiro de 2002 e trouxe consigo vários avanços, dentre eles, o incremento de recursos humanos e a reorganização do processo de trabalho das unidades básicas de saúde (UBS), tendo como eixos norteadores o acesso, o acolhimento, o vínculo e a resolutividade $\mathrm{e}^{2,3}$.

O acolhimento representa hoje uma política institucional implantada e aceita pelo grupo gerencial devido ao reconhecimento da humanização que a proposta agrega. Esta transformação do processo de trabalho nos serviços exige da equipe capacidade de distinguir problemas, riscos e agravos e adequar a resposta à complexidade dos problemas trazidos pelos usuários, remetendo a uma maior responsabilização dos profissionais ${ }^{4,5}$. Existe hoje uma diversificação da oferta de ações de promoção da saúde e implementação de uma série de atividades nas UBS, buscando uma abordagem integral dos usuários. Observa-se a ampliação da demanda espontânea, em especial para a população adulta, ultrapassando a lógica "programática" que excluía a entrada de usuários que não se enquadravam nos programas e prioridades estabelecidas. A Vigilância à Saúde tornou-se um referencial importante na organização do processo de trabalho das equipes, sendo caracterizada pela intervenção sobre problemas que exigem atenção e acompanhamento contínuos e articulação entre as ações de promoção, prevenção, cura e reabilitação, sob a ótica do conceito de risco ${ }^{6}$. 
A diversidade de demandas, motivos e características dos usuários que procuram o acolhimento requer diferentes respostas da equipe para a clientela. A atuação das ESF deve partir do diagnóstico e avaliação da área de abrangência, o que pode ser realizado a partir de instrumentos formais e informais. $\mathrm{O}$ acolhimento pode fornecer informações importantes para a compreensão das necessidades dos usuários e sobre a capacidade resolutiva da UBS, sendo, portanto, uma importante ferramenta para o planejamento das ações das equipes de saúde da família (ESF) 7 .

Este estudo teve por objetivo analisar a demanda não-programada de crianças e adolescentes de uma das ESF do Centro de Saúde São Marcos (CSSM), visando ao planejamento de suas ações. Conhecer e organizar a porta de entrada na UBS facilitará a abordagem e a organização local, bem como o monitoramento das ações desenvolvidas.

\section{Material e Métodos}

Este estudo foi desenvolvido no Centro de Saúde São Marcos, no período de outubro de 2005 a setembro de 2006. Os resultados apresentados dizem respeito ao acolhimento de usuários com menos de 20 anos de idade pertencentes à área da Equipe Azul.

O Centro de Saúde São Marcos está localizado na regional nordeste, é uma das 150 UBS de Belo Horizonte e é responsável por nove setores censitários, sendo oito classificados em médio risco e um em elevado risco, segundo critérios de vulnerabilidade. Possui população de aproximadamente 12 mil pessoas, $55 \%$ da população têm menos de quatro anos de estudo, 20\% dos domicílios possuem até três cômodos, os domicílios têm em média quatro moradores, $50 \%$ das famílias recebem até um salário mínimo e 442 famílias recebem bolsa-família ${ }^{8,9}$.

A população da área de abrangência está distribuída em três equipes de saúde da família, duas equipes de saúde bucal, profissionais de apoio (clínico geral, pediatra, ginecologista, enfermeira e assistente social), docentes e discentes dos cursos de graduação em medicina e em fisioterapia da UFMG, funcionários administrativos e gerente.

A Equipe Azul é composta por um médico, uma enfermeira, dois auxiliares de enfermagem e cinco agentes comunitários de saúde (ACS), cada uma responsável por uma microárea (MA 06, 07, 08, 09 e 10). O território das MA seis e oito é considerado de elevado risco, segundo critério de vulnerabilidade. $\mathrm{Na}$ área da equipe, existem 1163 famílias cadastradas com 4396 pessoas. Aproximadamente 97\% dos domicílios têm rede oficial de água, 94\% possuem rede de esgoto e $99 \%$, coleta de lixo ${ }^{9}$. As mulheres representam $52 \%$ da população. Quase 300 crianças têm menos de cinco anos, sendo que aproximadamente 80 delas têm menos de dois anos de idade. Os adolescentes representam 18\% da população da área de abrangência da Equipe Azul. Cerca de $70 \%$ das crianças com seis meses ou menos estão em aleitamento materno e são freqüentes ao acompanha- mento mensal de saúde. A cobertura vacinal em crianças menores de cinco anos é de $98 \%$. Apenas 5\% das crianças menores de cinco anos estão inscritas no Programa de Combate à Desnutrição. Existem atualmente 11 crianças recebendo medicação inalatória profilática para asma pelo programa Criança que Chia (consolidado dos registros da equipe).

O acolhimento no CSSM é realizado durante todo horário de funcionamento da unidade, embora cada equipe tenha um horário pré-definido, de conhecimento da comunidade, para atendimento da demanda espontânea. Esta primeira escuta é realizada pela enfermeira juntamente com uma auxiliar de enfermagem.

Foram analisados os registros do atendimento à demanda não-programada no horário reservado para a Equipe Azul, no período de outubro de 2005 a setembro de 2006. Os dados foram obtidos do impresso específico do acolhimento de uso diário, padronizado pela Secretaria Municipal de Saúde de Belo Horizonte, e dos livros de registros da Equipe Azul. A amostra foi composta por 30\% dos cerca de cinco mil atendimentos realizados, sorteados aleatoriamente por sistemática. O banco de dados foi analisado no Epi-Info versão 6.04b. O instrumento para coleta de dados continha questões sobre idade, sexo, microárea da residência, tipo de demanda, motivo da procura. 


\section{Resultados}

Foram analisados 1.602 atendimentos à demanda não-programada da Equipe Azul, distribuídos pelos 12 meses do período de estudo. A Tabela 1 mostra a distribuição dos atendimentos segundo a faixa etária dos usuários. Nota-se que os usuários com menos de 20 anos representaram 39,2\% ( $\mathrm{n}=627)$ dos atendimentos.

A freqüência de crianças do sexo masculino e feminino foi semelhante entre os menores de 10 anos; porém, entre os adolescentes, as mulheres corresponderam a 67,7\% dos atendimentos.

O Gráfico 1 apresenta a distribuição dos atendimentos de pacientes com menos de 20 anos por microárea (MA). Essa distribuição não foi homogênea, e a diferença de freqüência entre as microáreas mostrou-se estatisticamente significativa ( $p<0,01$; qui-quadrado).

A distribuição dos atendimentos segundo tipo de demanda dos usuários é apresentada no Gráfico 2. Cerca de $80 \%$ dos pacientes menores de 20 anos tinham queixas agudas. Os pacientes também demandaram a unidade para

Tabela 1. Distribuição dos usuários atendidos no acolhimento da Equipe Azul segundo a faixa etária, de outubro/2005 a setembro/2006, CSSM, Belo Horizonte (MG)

\begin{tabular}{lcc}
\hline \multicolumn{1}{c}{ Faixa etária } & $\mathbf{N}^{\mathbf{0}}$ & Percentual \\
\hline$\leq 2$ anos & 148 & $9,2 \%$ \\
$>2$ anos $\leq 10$ anos & 318 & $19,9 \%$ \\
$>2$ anos a $\leq 19$ anos & 161 & $10,1 \%$ \\
$>19$ anos a $\leq 40$ anos & 402 & $25,1 \%$ \\
$>40$ anos a $\leq 59$ anos & 340 & $34,0 \%$ \\
$\geq 60$ anos & 195 & $12,2 \%$ \\
Sem informação & 38 & $2,4 \%$ \\
Total & 1.602 & $100 \%$ \\
\hline
\end{tabular}

Gráfico 1. Distribuição das crianças e adolescentes atendidas no acolhimento da Equipe Azul, segundo a microárea (MA) de residência dos usuários, CSSM, Belo Horizonte, de outubro/2005 a setembro/2006.

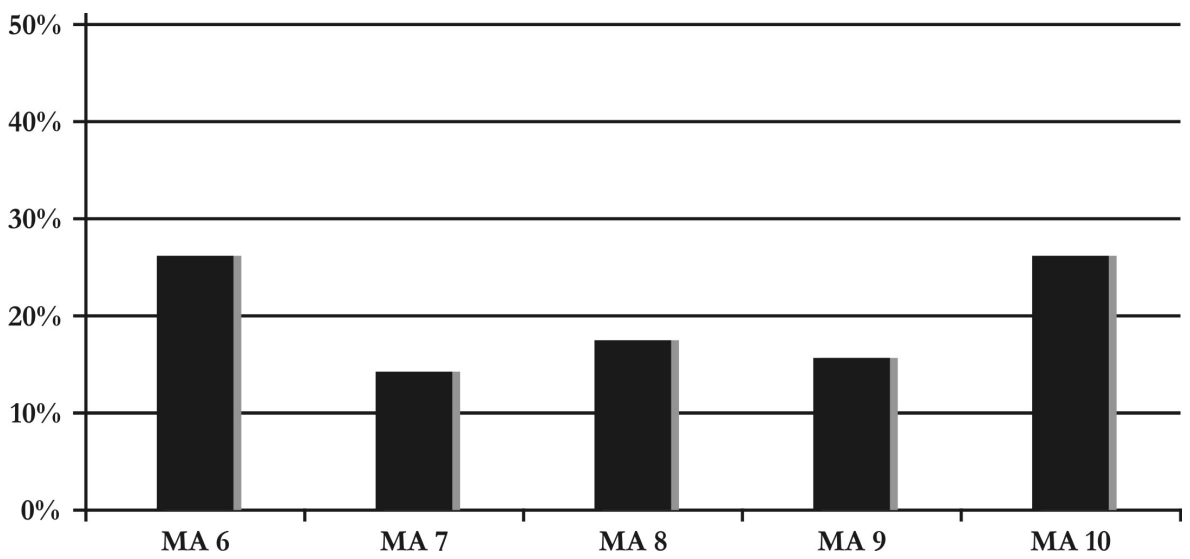


Gráfico 2. Distribuição das crianças e dos adolescentes atendidos no acolhimento da Equipe Azul, segundo o tipo de demanda dos usuários, CSSM, Belo Horizonte, de outubro/2005 a setembro/2006.



Gráfico 3. Distribuição das crianças e adolescentes atendidas no acolhimento da Equipe Azul e dos pacientes com queixas agudas, segundo a faixa etária, CSSM, Belo Horizonte, de outubro/2005 a setembro/2006.

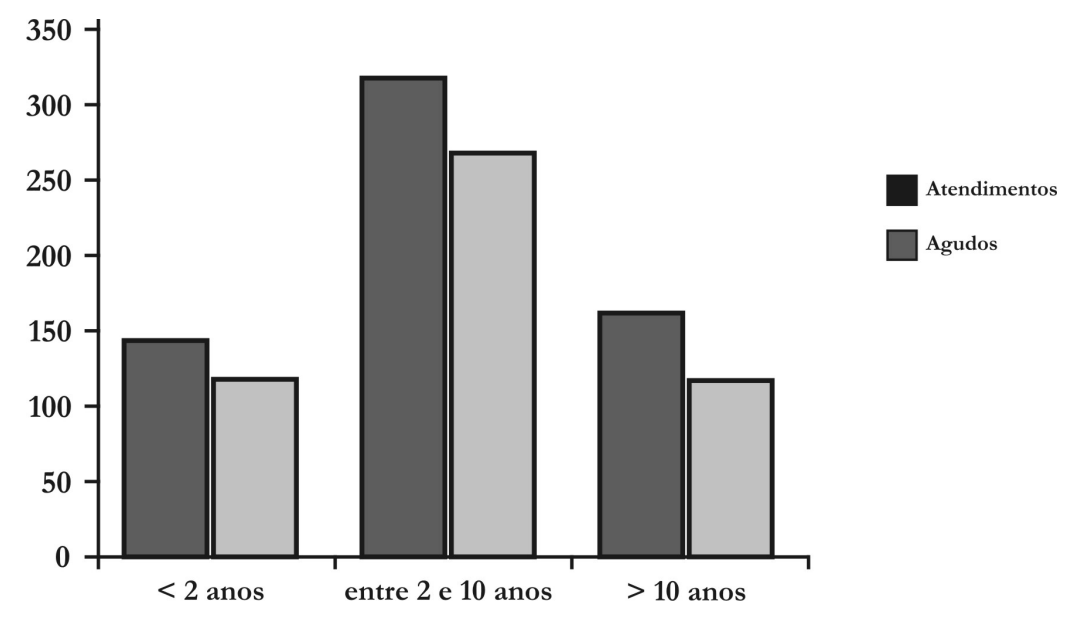

Gráfico 4. Distribuição das crianças e adolescentes atendidas no acolhimento da Equipe Azul, segundo o motivo de procura, CSSM/Belo Horizonte, de outubro/2005 a setembro/2006.

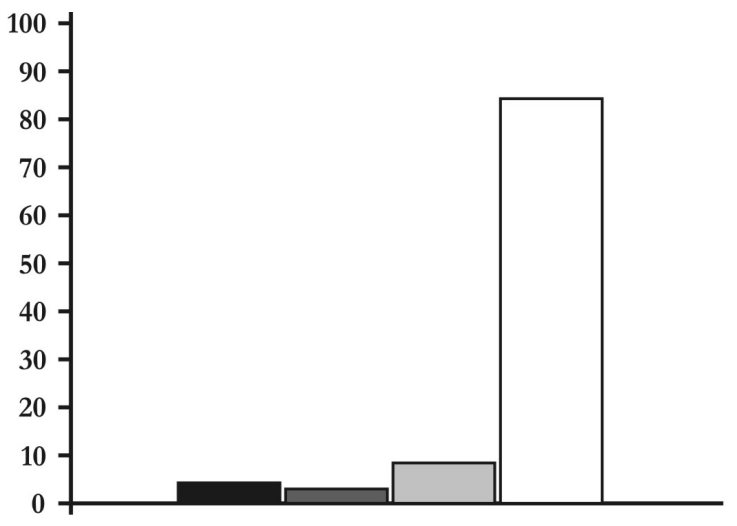

Puericultura

$\square$ GO

$\square$ Problemas respiratórios

$\square$ Outros motivos 
agendamento de consultas médicas e/ou para mostrar resultados de exames (16,9\%); trocar receitas e/ou pedir exames $(3,5 \%)$; pedir atestado médico $(0,5 \%)$ e por outros tipos de demanda (1,3\%). Cerca de 100 crianças procuraram o acolhimento para agendar consultas e/ou mostrar exames; dessas, $12 \%$ eram pacientes de puericultura, $2 \%$ procuravam consultas de saúde mental, $14 \%$ tinham queixas tocoginecológicas, 5\% eram portadores de problemas respiratórios crônicos, como asma, e $67 \%$ procuravam por consultas pediátricas.

A proporção de casos agudos em relação ao total de atendimentos nas crianças menores de dois anos, entre dois e dez anos e nos adolescentes é apresentada no Gráfico 3. A freqüência de pacientes com casos agudos nas diversas idades mostrou diferenças estatisticamente significativas $(\mathrm{p}=0,01$; qui-quadrado), sendo a proporção de casos agudos maior na faixa etária de dois a dez anos do que nas outras idades.

Quanto ao motivo de procura, $84,1 \%$ dos atendimentos foram classificados como "outros motivos", ou seja, não pertencem aos grupos prioritários como puericultura ou problemas respiratórios. $\mathrm{O}$ agendamento para ginecologia entre as adolescentes representou $12,8 \%$ do total dos atendimentos. Apenas quatro desses atendimentos foram para agendamento de pré-natal. Os motivos de procura foram estatisticamente diferentes quando analisados por faixa etária ( $p<0,01$; qui-quadrado), com maior proporção de problemas respiratórios na faixa de dois a dez anos do que nas demais e maior freqüencia de problemas tocoginecológicos entre as adolescentes, como era de se esperar.

\section{Discussão}

Os dados analisados neste estudo foram obtidos das fichas de acolhimento da Secretaria Municipal de Saúde (SMSA-BH) e dos livros de registros da equipe, o que limitou, em parte, a inclusão de novas variáveis e a criação de outras categorias de análise. Estes registros basearam-se na percepção do paciente de suas demandas e motivos de procura do serviço, o que nem sempre coincidiu com a percepção dos profissionais, fato que merece ser melhor estudado. No entanto, o banco de dados foi exaustivamente conferido pelos pesquisadores, tornando-o consistente e permitindo conclusões confiáveis.

O acolhimento de crianças e adolescentes foi responsável por cerca de $40 \%$ do total de atendimentos realizados pela equipe no período do estudo, o que representa um volume considerável de demandas para a equipe. Entre os menores de 20 anos, cerca de 51\% dos atendimentos foram para crianças entre dois e dez anos. Esses dados são preocupantes, pois a equipe não possui um programa de ações específico para essa faixa etária. Soma-se a isso a alta freqüência de pacientes com queixas agudas, especialmente entre as crianças de dois a dez anos. A ESF considera que as agendas devem ser organizadas para que $o$ atendimento aos casos agudos seja feito no mesmo dia. Esses dados também justificam a necessidade de um pediatra de apoio para a ESF na própria UBS e a capacitação permanente dos médicos de família para o atendimento da nosologia prevalente.

A procura do acolhimento para agendar consultas e/ou mostrar exames foi relativamente pequena nesta faixa etária. Poucas eram as crianças em puericultura e portadores de problemas respiratórios crônicos, como a asma. Estes dados sugerem que as crianças estão sendo acompanhadas regularmente, o que lhes garante o agendamento do retorno e promove o vínculo com a ESF. Em 2005, o Ministério da Saúde e a Secretaria Municipal de Saúde de Belo Horizonte assumiram o compromisso com a saúde integral da criança e a redução da mortalidade infantil. Por meio de documentos próprios afirmam que o foco das ações de toda a equipe, cada um dentro de sua missão profissional, deve ser o cuidado com a criança menor de cinco anos, em todas as oportunidades possíveis ${ }^{10,11}$. Na linha de cuidado sugerida pela SMSA-BH, o nascimento saudável, o crescimento/desenvolvimento e alimentação saudáveis, com enfoque prioritário para as crianças de maior risco e o cuidado do paciente com doenças prevalentes, são as ações que todas as ESF devem realizar plenamente ${ }^{11}$. No entanto, a faixa etária e as demandas mais freqüentes dos pacientes pediátricos parecem não pertencer aos grupos de atenção prioritários nem contempladas nas propostas 
de trabalho da ESF da UBS, o que exige uma reflexão da equipe sobre seu processo de trabalho.

Entre os adolescentes, a presença das mulheres foi marcante. No entanto, chama a atenção a pequena procura por agendamento para ginecologia. Este representou apenas 12,8\% desse tipo de atendimento. Apenas quatro desses atendimentos foram motivados por pré-natal. Existem várias especificidades no atendimento à adolescente. Com a progressiva antecipação do início da puberdade, a capacidade reprodutiva se instala mais cedo, assim como o início da atividade sexual e a ocorrência de doenças sexualmente transmissíveis. É necessária uma atenção especial em relação à ocorrência cada vez mais precoce do câncer de colo de útero ${ }^{12,13}$. Nota-se que os adolescentes, de um modo geral, não buscam informações ou assistência no serviço ou o serviço não está realmente preparado para oferecêlo. É necessário garantir estratégias de educação sexual e reprodutiva, saúde mental, prevenção de acidentes, na relação familiar e maus-tratos, voltadas para promoção da saúde e prevenção de doenças com maior risco de ocorrência nesta faixa etária.

O estudo mostrou diferenças significativas na freqüência de atendimentos entre as microáreas de abrangência, porém estas diferenças parecem não estar relacionadas à vulnerabilidade social. De acordo com a Prefeitura Municipal de Belo Horizonte, o índice de vulnerabilidade é construído levando-se em consideração a associação de variáveis socioeconômicas e ambientais, com a finalidade de evidenciar as desigualdades no perfil epidemiológico de grupos sociais distintos em determinadas áreas geográficas ${ }^{14}$. Nessas áreas, a assistência à população deve ser priorizada pelas ESF. A MA 06 e MA 08 têm maior índice de vulnerabilidade, porém a procura por atendimento pela população da MA 8 foi proporcionalmente menor do que das outras microáreas. A MA 07 é distante da unidade e o trajeto tem topografia acidentada. A distribuição dos atendimentos entre as microáreas da Equipe Azul remete à reflexão sobre o acesso. $\mathrm{O}$ acesso dos usuários envolve aspectos geográficos, a forma e o tempo de deslocamento da residência à UBS, entre outros. Esses aspectos são fatores dificultadores que explicam a baixa freqüência da população de algumas microáreas no acolhimento ${ }^{15}$. Atravessar grandes distâncias com crianças doentes e sem alternativas de transporte público pode inibir o acesso. Este fato torna-se ainda mais relevante por representar um risco a mais de adoecer e morrer para esta população ${ }^{16}$.

Outro aspecto a ser trabalhado dentro da equipe é o vínculo profissional/usuário. Para Merhy, o encontro entre esses sujeitos produz uma relação de escuta e responsabilização, a partir da qual se constituem os compromissos ${ }^{17}$. A responsabilização para com os problemas dos usuários vai além de um atendimento propriamente dito, diz respeito também ao vinculo necessário entre o serviço e a população ${ }^{18}$. A equipe percebe diferenças na atuação dos profissionais responsáveis por cada microárea, como a dificuldade em percorrer toda a área, deixando parte de sua população sem ligação com a UBS nas ações planejadas, o perfil do profissional, sua inserção na comunidade, entre outras. É possível que a qualidade do vínculo do ACS com os usuários possa estar relacionada com as diferenças do número de atendimentos entre algumas MA, visto que não há diferenças significativas no número de habitantes e no índice de vulnerabilidade.

Quando o motivo da procura da UBS foi analisado, observou-se que mais de $80 \%$ dos usuários tinham “outros motivos" que não o atendimento nos grupos prioritários como a puericultura, por exemplo. Compreender estes "outros motivos" é um desafio para a ESF, dada a sua complexidade e o volume de atendimentos que geram. Os motivos e demandas do usuário podem ser expressos explicitamente ou não, podendo refletir algum outro desejo ou necessidade em qualquer dimensão da sua existência ${ }^{7}$. Sendo assim, este grupo de crianças se torna uma verdadeira "caixa-preta" para a equipe, cuja compreensão pode ser determinante no planejamento de suas ações em todos os níveis. Esse dado indica também a necessidade da equipe contar com profissionais bastante preparados para lidar com a enorme variedade de problemas trazidos pelas crianças e adolescentes e um amplo cardápio de opções de abordagem destes pacientes. 
Por fim, é necessário refletir sobre o processo de acolhimento instituído no Centro de Saúde São Marcos. Desde 1994, existia uma orientação da SMSA-BH para que as UBS instituíssem o acolhimento como uma ação prioritária, que procurasse responder às dificuldades de acesso da população e, ao mesmo tempo, humanizar as relações entre os serviços/profissionais e usuários. Com essa implantação, houve grande incremento da capacidade operacional da rede básica, embora alguns problemas tivessem permanecido latentes, como o enfoque da integralidade, da resolutividade e da responsabilização².

Em 2003, num seminário para reorganização da Atenção Básica no Município de Belo Horizonte, pactuou com todas as UBS as novas diretrizes assistenciais do Programa BH-VIDA: Saúde Integral. Todos os Centros de Saúde foram orientados a viabilizar o acolhimento durante todo o seu horário de funcionamento. Com isso, a população encontrou portas abertas, e a falta de acesso à rede básica passou a ser um problema secundário. Atualmente, podem ser vistas inúmeras maneiras de funcionamento deste acolhimento no que se refere à formatação das equipes que recebem o usuário, das atividades que oferecem e do cardápio de respostas. Há um grande incômodo do trabalhador com o dispositivo do acolhimento, que alia a incompreensão com as potencialidades do dispositivo à grande demanda de usuários nas UBS, com problemas das mais diversas naturezas, como observado neste estudo. Há ainda a dificuldade de lidar com a demanda não imediatamente reconhecida como da saúde, o que provoca uma sensação de pouca resolutividade por parte das equipes. As equipes relatam dificuldades em realizar uma "escuta qualificada", que exige atenção e disponibilidade. Os principais entraves encontrados são a demanda volumosa, o despreparo dos trabalhadores, o predomínio da "lógica médica" na prática dos profissionais e as expectativas da sociedade ${ }^{2}$.

Todas essas questões estão presentes no dia-a-dia da Equipe Azul do Centro de Saúde São Marcos, assim como de muitas ESF pelo país a fora e, certamente, influenciaram os resultados aqui apresentados. A partir do mo- mento em que o acesso e acolhimento garantam a entrada do usuário no sistema de saúde, o desafio é promover uma ordenação dos fluxos, para que as ações das equipes se tornem mais resolutivas e contribuam para a melhoria da situação de saúde da população.

\section{Conclusões}

A realização deste estudo mostrou a importância de se analisar a demanda não-programada nas ESF com vistas ao planejamento de suas ações. Com metodologia simples e envolvendo todos os membros da ESF, conseguiu revelar as necessidades e os motivos de procura dos usuários, indicando pontos de estrangulamento nas agendas dos profissionais, carência de ações preventivas e de promoção da saúde, como a pequena procura por ginecologia entre as adolescentes, além de ter demonstrado a boa cobertura de programas como a puericultura e o da Criança que Chia.

É preciso trabalhar para que o acesso se torne mais inclusivo, permitindo que o usuário conheça a estrutura do serviço, da rede assistencial e se sinta capaz de influenciar em seu andamento. Existe a necessidade de qualificar a relação trabalhador/usuário, uma vez que esta relação não ocorre de forma uniforme entre os componentes da ESF. A equipe deve investir de forma diferenciada nas microáreas de risco elevado procurando desvendar as necessidades não expressas no estudo e direcionar ações em saúde. A equipe deve rediscutir sua agenda a partir do conhecimento de sua realidade, definindo as prioridades, expandindo seu cardápio de ações e traçando um plano de ação com base nos resultados deste estudo. A organização do processo de trabalho deve propiciar o deslocamento do eixo centrado no médico para uma equipe multiprofissional equipe de acolhimento - que se encarrega da escuta do usuário, comprometendo-se em resolver seus problemas.

Finalmente, o Centro de Saúde São Marcos deve definir uma agenda de estudos para a compreensão da demanda não-programada nas outras equipes e para monitoramento das ações implantadas a partir desses resultados na Equipe Azul, bem como procurar captar as 
demandas não expressas dos usuários e a satisfação deles com as mudanças no processo de trabalho das equipes.

\section{Referências}

1. Brasil. Ministério da Saúde. Secretaria de Atenção à Saúde. Núcleo técnico de Política Nacional de Humanização. Monitoramento e avaliação na política nacional de humanização na rede de atenção e gestão do SUS: manual com eixos avaliativos e indicadores de referências. Brasilia (DF): Ministério da Saúde; 2006.

2. Associação Mineira de Medicina de Família e Comunidade. $\mathrm{O}$ acolhimento nas unidades de atenção básica de Belo Horizonte. Proceedings of Ciclos de debates da Associação Mineira de Medicina de Família e Comunidade; agosto 2006; Belo Horizonte, Brasil. Disponível em: http:// www.smmfc.org.br/gesf/ACOLHIMENTOAMMFC.ppt. Acesso em 02 fev. 2008.

3. Merhy EE. Em busca da qualidade dos serviços de saúde: os serviços de porta aberta para a saúde e o modelo tecnoassistencial em defesa da vida. In: Cecílio LC, Organizador. Inventando a mudança em saúde. São Paulo: Hucitec; 1994. p.116-60.

4. Brasil. Ministério da Saúde. Secretaria-Executiva. Núcleo Técnico da Política Nacional de Humanização. HumanizaSUS: acolhimento com avaliação e classificação de risco: um paradigma ético-estético no fazer em saúde. Brasilia (DF): Ministério da Saúde; 2004.

5. Malta DC. Buscando novas modelagens em saúde, as contribuições do projeto Vida e Acolhimento para a mudança do processo de trabalho na rede pública de Belo Horizonte [Tese]. Campinas (SP): Universidade Estadual de Campinas; 2001.

6. Paim JS. Vigilância da saúde: dos modelos assistenciais para promoção da saúde. In: Czeresnia $\mathrm{D}$, organizadora. Promoção da saúde: conceitos, reflexões, tendências. Rio de Janeiro: Ed. Fiocruz; 2003. p. 161 - 74.

7. Belo Horizonte. Secretaria Municipal de Saúde. A atenção básica de saúde em Belo Horizonte: recomendações para a organização local. Belo Horizonte: Secretaria de Saúde; 2006.

8. Brasília. Fundação Instituto Brasileiro de Geografia e
Estatística. Censo 2000. Brasilia: Fundação Instituto Brasileiro de Geografia e Estatística. 2000. Disponível em http:// www.ibge.gov.br/censo. Acesso em 02 fev. 2008.

9. Belo Horizonte. Secretaria de Saúde. Sistema de listagem e tabulação de dados on-line/intranet/PBH. Cadastro Social. Disponível em: http://www.carai.pbh/sqltab/ loginlistasqe.asp. Acesso em 01 apr. 2008.

10. Brasil. Ministério da Saúde. Secretaria de Atenção a Saúde. Departamento de Ações Programáticas Estratégicas. Agenda de compromissos para a saúde integral da criança e redução da mortalidade infantil. Brasília (DF): Ministério da Saúde; 2005.

11. Belo Horizonte. Secretaria Municipal de Saúde. Coordenação de Atenção Básica. BH-Viva Criança: Agenda de compromissos pela saúde integral da criança e adolescente e redução da mortalidade infantil. Belo Horizonte (MG): Secretaria Municipal de Saúde; 2003.

12. Brasil. Secretaria de Atenção à Saúde. Departamento de Atenção Básica. Caderno de Atenção Básica: controle dos cânceres do colo do útero e da mama. Brasília(DF): Ministério da saúde; 2006.

13. Alves CR, Alvim CG, Magalhães ME, Almeida JS, Goulart LM, Dias LS, et al. Saúde da criança e do adolescente: políticas públicas. In: Alves CR, Viana MR, organizadores. Saúde da Família: cuidando de crianças e adolescentes. Belo Horizonte (MG): Editora Coopmed; 2006. p. 7-14.

14. Belo Horizonte. Secretaria de Saúde. Gerência de Epidemiologia e Informação. Índice de Vulnerabilidade a Saúde 2003 (risco). http://www.pbh.gov.br/smsa/ biblioteca $/$ mostraarquivo.php?documento $=339$ Acesso em 01/04/2008.

15. Ramos DD, Lima MA. Acesso e acolhimento aos usuários em uma unidade de saúde de Porto Alegre, Rio Grande do Sul, Brasil. Cad Saúde Pública 2003; 19 (1): 27-34.

16. Starfield B. Atenção primária: equilíbrio entre necessidades de saúde, serviços e tecnologia. Brasília(DF): UNESCO, Ministério da Saúde; 2002.

17. Merhy EE. Em busca do tempo perdido: a micropolítica do trabalho vivo em saúde .In: Merhy EE, 
Onocko RC organizadores. Agir em saúde: um desafio para o publico. São Paulo: Hucitec; p. 71-112.

18) Solla JJ. Acolhimento no sistema municipal de saúde.

Rev Bras Saúde Matern Infant 2005; 5 (4): 493 - 503.

\section{Endereço para Correspondência:}

Av. Prof. Alfredo Balena, $190 / 4^{\circ}$ andar

Departamento de Pediatria

Belo Horizonte - MG

CEP: $30.130-100$

\section{Endereço eletrônico:}

lindgren@medicina.ufmg.br 